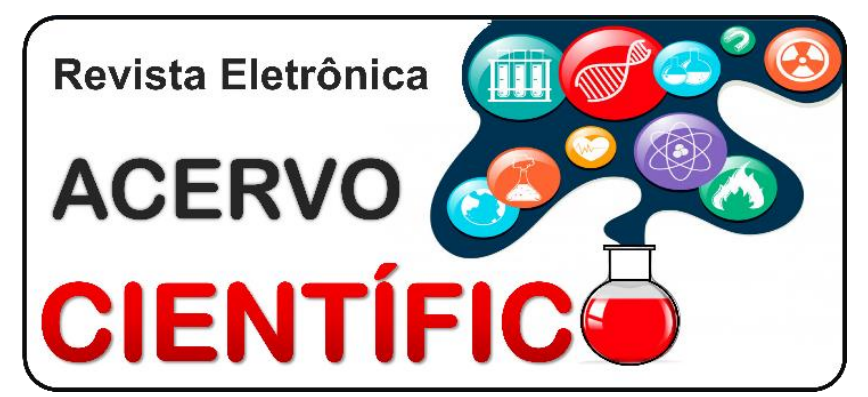

REVISÃO BIBLIOGRÁFICA

Recebido em: 12/2019

Aceito em: $1 / 2020$

Publicado em: 9/2020

\title{
Microagulhamento associado ao drug delivery no tratamento do melasma feminino
}

\author{
Microneedle associated to the drug delivery in the treatment of female melasma
}

Microagulación asociada a la entrega de drogas en el tratamiento del melasma femenino Joseni da Visitação Coelho ${ }^{1 *}$, Ana Paula Mendes Geitenes ${ }^{1}$.

\begin{abstract}
Resumo: O melasma é hiperpigmentação crônica adquirida, acometem áreas fotoexpostas da pele como face e eventualmente colo e braços. Sendo sua etiologia multifatorial, a patogenia ainda permanece desconhecida, sabendo-se que a radiação ultravioleta é o fator preponderante. O microagulhamento ou Indução Percutânea de Colágeno por Agulhas (IPCA) foi recentemente incluído como abordagem desta patologia. Assim, buscou-se reunir e discutir dados científicos que comprovem a eficácia do microagulhamento associado ao Drug delivery no tratamento do melasma feminino. Foi realizada uma revisão da literatura, nas principais bases de dados virtuais, Pubmed, Lilacs, Medline, Scielo e Bireme, de artigos publicados entre 2009 e 2019, que abordassem o tema. Demonstrou-se a eficácia da combinação das técnicas descritas no clareamento da pele, na hidratação e auxiliando no rejuvenescimento, porém devido a pequena quantidade de estudos, se faz necessário mais publicações para melhor elucidação dos dados.
\end{abstract}

Palavras-chave: Terapia combinada, Melasma, Administração cutânea.

\begin{abstract}
Melasma is acquired chronic hyperpigmentation, affecting photoexposed areas of the skin such as the face and eventually the neck and arms. Since its etiology is multifactorial, the pathogenesis remains unknown, knowing that ultraviolet radiation is the predominant factor. Microneedling or Percutaneous Needle Collagen Induction (IPCA) was recently included as an approach to this pathology. Thus, we sought to gather and discuss scientific data that prove the effectiveness of microneedling associated with Drug delivery in the treatment of female melasma. A literature review was carried out in the main virtual databases, Pubmed, Lilacs, Medline, Scielo and Bireme, of articles published between 2009 and 2019, which addressed the topic. The effectiveness of the combination of the techniques described in skin whitening, hydration and aiding rejuvenation has been demonstrated, however due to the small amount of studies, more publications are needed to better clarify the data.
\end{abstract}

Keywords: Combination therapy, Melasma, Cutaneous administration.

Resumen: El melasma se adquiere hiperpigmentación crónica, que afecta a las áreas fotoexpuestas de la piel, como la cara y, finalmente, el cuello y los brazos. Dado que su etiología es multifactorial, la patogénesis

${ }^{1}$ Centro Universitário do Brasil (UNIRB), Salvador - Bahia.

*E-mail: joseniunirb@gmail.com 
sigue siendo desconocida, sabiendo que la radiación ultravioleta es el factor predominante. La microinyección o la inducción percutánea de colágeno con aguja (IPCA) se incluyó recientemente como un enfoque para esta patología. Por lo tanto, buscamos reunir y discutir datos científicos que prueben la efectividad de las microagujas asociadas con la administración de fármacos en el tratamiento del melasma femenino. Se realizó una revisión de la literatura en las principales bases de datos virtuales, Pubmed, Lilacs, Medline, Scielo y Bireme, de artículos publicados entre 2009 y 2019, que abordaron el tema. Se ha demostrado la efectividad de la combinación de las técnicas descritas en el blanqueamiento de la piel, la hidratación y el rejuvenecimiento, sin embargo, debido a la pequeña cantidad de estudios, se necesitan más publicaciones para aclarar mejor los datos.

Palabras clave: Terapia combinada, Melasma, Administración cutánea.

\section{INTRODUÇÃO}

O melasma é uma hiperpigmentação crônica adquirida, de coloração acastanhada clara ou escura geralmente simétrica, também conhecido como dermatose crônica colorida, acometem áreas fotoexpostas da pele como face e eventualmente colo e braços. O melasma é incidente em todos os fototipos de pele e em ambos os sexos embora predominante em mulheres em idade fértil (SARAIVA LPPG, et al., 2018).

A causa do melasma apresenta vários fatores desencadeantes sendo que o modo de desenvolvimento ou de evolução é desconhecida, embora a exposição ao sol é o fator mais dominante, uma vez que fisiologicamente pode causar peroxidação dos lipídios nas membranas celulares, formando radicais livres que podem estimular os melanócitos a produzir excesso de melanina. Outros fatores predisponentes são o uso de contraceptivos orais, tratamento hormonal, gravidez e história familiar (PURIM MSK e AVELAR SFM, 2012).

No tratamento do melasma são utilizados despigmentante e antioxidantes tópicos e orais, peeling químicos, tecnologias como: microagulhamento, laser de Q-Swchited 1064, em alguns estudos demonstram o pulse dye laser como opção para o melasma com competente vascular predominante (SARAIVA LPPG, et al., 2018).

$\mathrm{Na}$ Dermatologia a via mais utilizada para administração de medicamentos é a via cutânea devido os inúmeros benefícios em relação às vias sistêmicas principalmente para minimizar os efeitos colaterais e manter a adesão do paciente. Mesmo que a epiderme apresente uma bicamada lipídica do estrato córneo, sendo a principal barreira para a administração de medicamentos aplicados sobre a pele, ainda assim é a via de escolha para os tratamentos de pele. Sendo assim a técnica denominada Drug delivery consiste em facilitar essa penetração cutânea, através de microperfurações sendo realizada a aplicação de medicações que irá ter uma resposta de absorção na pele satisfatória (CÉLIA K, et al., 2016).

O microagulhamento surgiu na década de 1990 na Alemanha, porém apenas em 2006 ficou conhecida pelo mundo. Trata-se de um equipamento com um rolo coberto por agulhas finas de aço inoxidável cirúrgico ou liga de titânio, as quais apresentam diversos comprimentos de diâmetro (KLAYN AP, et al., 2013; LIMA EVA, et al., 2013).

O microagulhamento tem como mecanismo de ação uma lesão controlada induzida por agulhas que produzem puncturas com 1 a 1,5 milímetros de profundidade, realizadas na pele. Podendo ser indicada como terapêutica com associação com técnica Drug Delivery a qual permite a liberação de fatores de crescimento que vai incentivar a produção de colágeno e elastina na derme papilar. Além de potencializar a permeação de princípios ativos despigmentante destinado ao clareamento, o qual estão ligados a interferência na produção de melanina (KLAYN AP, et al., 2013; LIMA EVA, et al., 2013).

Podendo ser realizado da forma tradicional utilizando cilindros contendo agulhas, conhecidos como rollers, ou através de uma técnica descrita recentemente conhecida como Microinfusão de Medicamentos pela Pele (MMP) realizada através da infusão de medicamentos (Drug delivery) utilizando o microagulhamento (ARBACHE S, ODOY CE, 2013). 
A regeneração da pele é rápida e os efeitos colaterais do microagulhamento são mínimos se comparada a outras técnicas ablativas, uma vez que provoca que a pele fique mais densa e resistente. É considerada de baixo custo/benefício quando comparada as tecnologias avançadas dessa área, apresenta resultados satisfatórios nas diversas alterações estéticas, não só ativando a circulação local, como também na melhora do aspecto geral do tecido. Podem apresentar resultados logo na primeira aplicação, assim a quantidade de sessões varia de acordo com o comprometimento estético e a condição clínica de cada indivíduo (PIATTI IL, 2013; LIMA EA, et al., 2013).

Assim o objetivo deste estudo foi reunir e discutir dados científicos que comprovem a eficácia do microagulhamento associado a Drug Delivery no tratamento do melasma feminino, com proposta de clareamento, hidratação, auxiliando no rejuvenescimento e consequentemente melhorando a qualidade de vida e o relacionamento interpessoal, assim como, o bem-estar e a auto estima.

\section{MÉTODOS}

O trabalho desenvolvido trata-se de uma revisão de literatura sobre o Microagulhamento associado a Drug Delivery no tratamento do melasma feminino. A busca bibliográfica foi desenvolvida por meio de informações em bibliotecas virtuais: Literatura Latino-Americana e do Caribe em Ciências da Saúde (Lilacs), Literatura Internacional em Ciências da Saúde (Medline), Scientific Electronic Library Online (Scielo) e Centro Latino-Americano e do Caribe de Informação em Ciências da Saúde (Bireme) com as seguintes palavras-chave: Terapia combinada; Melasma; Administração cutânea, utilizadas de forma combinada, traduzidas para o inglês e espanhol.

Foram incluídos artigos científicos, dissertações e teses que abordassem o tema proposto, realizados em humanos, com amostra populacional feminina para intervenção e que utilizassem o microagulhamento associado a infusão de medicamento como técnica de tratamento, publicados entre 2009 a 2019 nos idiomas português e inglês. Produções não disponíveis gratuitamente na íntegra e de forma virtual foram excluídas. Para acesso ao texto completo, foram usados os seguintes recursos: link disponível diretamente nas bases de dados, busca no portal do periódico em que o artigo, dissertação ou tese foi publicada, busca no portal Coordenação de Aperfeiçoamento de Pessoal de Nível Superior (CAPES).

A análise das informações foi realizada por meio de leitura exploratória e analítica do material encontrado. Esta seleção se baseia nos títulos da abordagem como ideia principal, o microagulhamento associado a Drug Delivery no tratamento do melasma feminino. Ao final da busca, foram excluídos os títulos repetidos e os que não se aplicam ao tema escolhido. Em seguida foi feita a leitura detalhada dos resumos dos artigos a fim de selecionar aqueles que abordassem exclusivamente o presente tema.

\section{RESULTADO E DISCUSSÃO}

Foram selecionados 8 estudos, sendo utilizados 6 (Quadro 1), que se encaixaram nos critérios de inclusão. Os demais estudos não retratavam a proposta do atual trabalho, sendo, portanto, excluídos. 
Quadro 1 - Organização dos artigos pesquisados, ordenados por ano de publicação.

\begin{tabular}{|c|c|c|c|}
\hline Título/autor(es)/ano & Objetivos & Tipo de estudo & Principais achados \\
\hline $\begin{array}{l}\text { Microagulhamento: } \\
\text { Estudo experimental e } \\
\text { classificação da injúria } \\
\text { provocada (LIMA EVA, } \\
\text { et al., 2013). }\end{array}$ & $\begin{array}{l}\text { Estabelecer a relação } \\
\text { do comprimento das } \\
\text { agulhas dos cilindros } \\
\text { utilizado para o } \\
\text { microagulhamento, com } \\
\text { a profundidade do dano. }\end{array}$ & $\begin{array}{c}\text { Estudo } \\
\text { Experimental }\end{array}$ & $\begin{array}{l}\text { O microagulhamento pode ser } \\
\text { indicado com o objetivo de } \\
\text { estimular produção de } \\
\text { colágeno. A relação entre o } \\
\text { comprimento da agulha } \\
\text { utilizada e dano provocado na } \\
\text { pele facilita a escolha do } \\
\text { instrumento nas diferentes } \\
\text { indicações. }\end{array}$ \\
\hline $\begin{array}{l}\text { Microagulhamento em } \\
\text { melasma facial } \\
\text { recalcitrante: uma série } \\
\text { de } 22 \text { casos (LIMA EA, } \\
\text { 2015). }\end{array}$ & $\begin{array}{c}\text { Utilizar o } \\
\text { microagulhamento, } \\
\text { como técnica isolada, } \\
\text { como opção terapêutica } \\
\text { nos casos de melasma } \\
\text { recalcitrante }\end{array}$ & Série de casos & $\begin{array}{l}\text { Pode-se presumir que o } \\
\text { clareamento substancial } \\
\text { observado na tonalidade do } \\
\text { grupo foi alcançado pelas } \\
\text { modificações ocorrida na pele } \\
\text { após a injúria. }\end{array}$ \\
\hline $\begin{array}{l}\text { Microagulhamento: } \\
\text { série de casos } \\
\text { associados Drug } \\
\text { Delivery (KALIL C, et } \\
\text { al., 2017) }\end{array}$ & $\begin{array}{l}\text { Descrevemos o uso do } \\
\text { microagulhamento } \\
\text { associado ao Drug } \\
\text { delivery no tratamento } \\
\text { de estrias e no } \\
\text { rejuvenescimento da } \\
\text { pele da face e mãos. }\end{array}$ & Série de casos & $\begin{array}{c}\text { Os resultados revelaram } \\
\text { melhora das manchas e textura } \\
\text { da pele na face, das mãos. O } \\
\text { estudo apresentou resultados } \\
\text { promissores associando } \\
\text { microagulhamento e Drug } \\
\text { delivery para tratamentos } \\
\text { dermatológicos. }\end{array}$ \\
\hline $\begin{array}{c}\text { Uso da técnica de } \\
\text { indução percutânea de } \\
\text { colágeno no tratamento } \\
\text { da hiperpigmentação } \\
\text { pós- inflamatória. } \\
\text { (TAGLIOLATTO S, et } \\
\text { al., 2017). }\end{array}$ & $\begin{array}{l}\text { Descreve-se a } \\
\text { experiência dos autores } \\
\text { em um caso desse tipo } \\
\text { de hipercromia. }\end{array}$ & $\begin{array}{l}\text { Artigos de revisão } \\
\text { com notas } \\
\text { técnicas do autor }\end{array}$ & $\begin{array}{l}\text { Com base na revisão de } \\
\text { literatura e em nossa } \\
\text { experiência clínica sugerimos o } \\
\text { microagulhamento, possa ser } \\
\text { incluído no tratamento da } \\
\text { hiperpigmentação pós- } \\
\text { inflamatória. }\end{array}$ \\
\hline $\begin{array}{l}\text { Tratamento de } \\
\text { melasma facial com } \\
\text { associação do } \\
\text { Microagulhamento } \\
\text { Robótico e Drug } \\
\text { Delivery de ácido } \\
\text { tranexâmico (SARAIVA } \\
\text { LPPG, et al., 2018). } \\
\end{array}$ & $\begin{array}{l}\text { Avaliar a melhora clínica } \\
\text { de melasma tratados } \\
\text { com microagulhamento } \\
\text { robótico associado a } \\
\text { Drug delivery de ácido } \\
\text { tranexâmico. }\end{array}$ & $\begin{array}{c}\text { Estudo } \\
\text { intervencionista }\end{array}$ & $\begin{array}{l}\text { Dos } 15 \text { pacientes de fototipos } \\
\text { de II a VI; } 31,11 \% \\
\text { apresentaram em sua maioria } \\
\text { melhora importante. A } \\
\text { associação proposta } \\
\text { apresentou resultados } \\
\text { satisfatórios para o tratamento } \\
\text { do melasma. }\end{array}$ \\
\hline $\begin{array}{l}\text { Avaliação histológica da } \\
\text { redução do conteúdo } \\
\text { melânico cutâneo após } \\
\text { realização d } \\
\text { e microagulhamento na } \\
\text { região anterior do colo } \\
\text { (PITASSI LHU, et al., } \\
\text { 2019). }\end{array}$ & $\begin{array}{l}\text { Avaliar a resposta } \\
\text { histológica cutânea após } \\
\text { o microagulhamento } \\
\text { para tratamento de } \\
\text { discromias }\end{array}$ & Série de casos & $\begin{array}{c}\text { O mecanismo do } \\
\text { microagulhamento para } \\
\text { promover clareamento, inclui } \\
\text { proliferação de fibroblastos e } \\
\text { neocolagênese na derme } \\
\text { superior. Esse é o primeiro } \\
\text { estudo a avaliar } \\
\text { histologicamente os achados } \\
\text { associados ao clareamento. }\end{array}$ \\
\hline
\end{tabular}

Fonte: COELHO JV, GEITENES APM, 2019. 
O melasma é doença dermatológica facilmente diagnosticada ao exame clínico, porém, apresenta uma cronicidade característica, com recidivas frequentes, grande refratariedade aos tratamentos existentes e ainda muitos aspectos fisiopatológicos desconhecidos; patogênese pouco estudada ainda; porém, sabe-se que alguns fatores podem influenciar no seu aparecimento, como radiação ultravioleta, predisposição genética, fatores hormonais, uso de contraceptivos orais, terapia de reposição hormonal, cosméticos, drogas fototóxicas e fotoalérgicas e fatores nutricionais (PURIM MSK, AVELAR SFM, 2012).

Vale destacar que o melasma é uma doença dérmica crônica e inestética da qual é responsável por uma grande procura aos tratamentos estéticos para solucionar essa pigmentação. Talvez, isso se explique por ser considerada em alguns casos desfigurante e pelos efeitos emocionais e psicológicos nos indivíduos portadores desse problema, os quais, muitas vezes, em virtude a essa insatisfação com a aparência, acabam comprometendo o convívio social.

O tratamento do melasma têm como objetivo o clareamento e melhorar o aspecto da pigmentação, sendo o resultado mais rápido quando a pigmentação se localiza mais superficial na pele; no caso de pacientes crônicos ou em estágio avançado de melasma a terapêutica pode ser logo e difícil penetração dos ativos despigmentantes na pele. Entretanto, o tratamento com o uso de microagulhamento associado aos ativos que são efetivos no tratamento do melasma (SILVEIRA LL, 2017; SILVA SN, 2018).

O microagulhamento é uma técnica recente que teve seu primeiro estudo-piloto no tratamento do melasma publicado em 2011, pela Revista Internacional Cirurgia Plástica, utilizando em uma hemiface uma medicação clareadora isolada tópica e a outra hemiface o uso com o microagulhamento associado a mesma substância. Foi realizada uma comparação fotográfica demonstrou-se que a hemiface com microagulhamento apresentou melhora expressiva em relação ao clareador tópico isoladamente, percebese, assim que quando ultrapassamos a barreira da bicamada lipídica da epiderme temos uma absorção da medicação tópica clareadora que agindo de forma mais expressiva (FABBROCINI G, et al., 2011).

A partir daí outros estudos foram realizados estabelecendo protocolos de tratamento com uso isolado do microagulhamento ou associado a ativo tópico. Considerando os resultados satisfatórios em 100\% dos pacientes no clareamento das manchas. Decorrente desses estudos, o microagulhamento tem se apresentado como tratamento minimamente invasivo para promover melhora clínica e histológica do melasma, demostrando a sua eficácia no tratamento (LIMA EA, 2015; TAGLIOLATTO S, et al., 2017).

A proposta de Saraiva LPPG, et al. (2018) é analisar se é eficiente o microagulhamento robótico associada ao Drug delivery de ácido tranexâmico, sendo que o ácido tranexâmico é um despigmentantes seguro e com baixo índice de efeitos colaterais, sendo utilizado em todos os tipos de fototipos e em período curto de tempo. Essa técnica apresenta uma resposta terapêutica satisfatória, uma vez que esse tratamento permite a intensificação do efeito local da medicação, quando utilizado com $2 \mathrm{~mm}$ de profundidade na pele.

Assim confirma-se com Kalil C, et al. (2017) que a associação do microagulhamento com o Drug delivery tem-se mostrado benéfica pois potencializa os resultados de ambas as técnicas. A perda controlada da integridade cutânea, que inicia o processo de cicatrização e culmina com a formação de colágeno tipo I, tem efeito no rejuvenescimento cutâneo e na melhora de cicatrizes. A associação de uma fórmula cosmética específica com o microagulhamento potencializa os mecanismos de ação sobre a síntese de melanina, sendo responsável pelo clareamento da face.

Estudos em pacientes com melasma resistente ao clareamento tópico, tratados com microagulhamento, demonstraram que podem ocorrer clareamento do melasma, e com relação aos estudos que analisaram histologicamente esses efeitos, foi evidenciado espaçamento e redução da pigmentação de melanina epitelial e densificação de colágeno da derme superior (LIMA EA, 2015; KALIL C, et al., 2017; SARAIVA LPPG, ET AL., 2018; PITASSI LHU, ET AL., 2019).

Os efeitos histológicos propostos pelo microagulhamento para o tratamento do melasma, ocasiona proliferação de fibroblastos e renovação de colágeno. Essas respostas restauram o dano a membrana basal e na porção superior da derme, evitando o contato dos melanócitos com estímulos melanogênicos dérmicos. Além disso, uma vez que o microagulhamento causa espessamento epidérmico, o procedimento 
provocaria proteção adicional a radiação UV. Além de estimular a formação de colágeno, a técnica que perfura camadas de pele com agulhas pequeninas melhora sua vascularização e a ação dos melanócitos (células produtoras de melanina, responsáveis pela pigmentação cutânea), diminuindo gradativamente as manchas do melasma (LIMA EA, 2015; PITASSI LHU, et al., 2019).

Lima EA (2015) e Pitassi LHU, et al. (2019) em seus estudos relatam que os processos de trauma tecidual podem ser classificados de acordo com a profundidade de perfuração das microagulhas: em leve (com agulhas de 0,25 a $0,5 \mathrm{~mm}$ ) e é indicada em quadros de rugas finas e brilho excessivo; moderado (com agulhas de 1,0 a 1,5mm), é aplicada em pacientes que apresentam flacidez cutânea, rugas médias, envelhecimento e melasma; e o profundo (com agulhas de 2,0 a 2,5mm) é indicada em pacientes que possuem estrias e cicatrizes.

Os exames microscópicos avaliaram que agulhas de $0,5 \mathrm{~mm}$ de comprimento de perfuração acometem até a derme papilar, já as agulhas de $2,5 \mathrm{~mm}$ de comprimento além da derme papilar se estende até a derme reticular. A quantidade de hemorragia, ou mesmo sangramento gerado foi proporcional ao aumento do comprimento das agulhas, sem apresentar lesão no tecido celular subcutâneo. Pacientes com melasma recalcitrante foram tratados com microagulhamento com agulhas no comprimento de $1,5 \mathrm{~mm}$ a qual foi capaz de provocar clareamento das manchas. E também, essas mesmas agulhas são usadas para 0 remodelamento de colágeno (LIMA EVA, et al., 2013; PITASSI LHU, et al., 2019).

O uso do microagulhamento em tratamentos estéticos possuem inúmeros efeitos, tais como a estimulação de colágeno sem promover dano tecidual. Assim a cicatrização ocorre rapidamente e os efeitos colaterais são mínimas. Já as desvantagens estão relacionadas com à capacitação profissional e ao treinamento específico, pois, dependendo da profundidade de perfuração atingida com a agulha, é necessário um tempo maior de recuperação da paciente; portanto, é necessário que o profissional realize uma avaliação cautelosa a fim de se evitar falsos resultados (LIMA EVA, et al., 2013).

Conforme Lima EA (2015) e Kalil C, et al. (2017) relatam que um dos efeitos do microagulhamento é potencializar a permeação de ativos, uma vez que os microcanais realizados com as perfurações das microagulhas facilitam a absorção, aumentando a penetração em até $80 \%$. Sendo assim, pode-se afirmar que a ação combinada do microagulhamento e de ativos cosméticos pode potencializar os resultados desejados.

O microagulhamento é um tratamento inovador e os efeitos da técnica associados a diferentes ativos proporcionam o rompimento das fibras de colágeno antigas, aumentando a renovação e crescimento de novas fibras proporcionando para a pele uma aparência saudável e mais uniforme. As vantagens de cada ativo usado individualmente demonstram que é fundamental a associação de produtos para a estimulação e formação de novo colágeno (SARAIVA LPPG, et al., 2018; PITASSI LHU, et al., 2019).

Conforme os estudos com o uso do microagulhamento pode aumentar a permeabilidade cutânea por aproximadamente 48 horas; esse tempo pode ser dilatado com a oclusão, que retarda a restauração do estrato córneo, considerando-se ainda que as combinações de substâncias podem apresentar natureza anidra e hidrorrepelente. Outros fatores que afetam a permeabilidade cutânea são as propriedades das drogas (ionização, concentração, lipossomas ou nanopartículas), a presença de cossolventes, o pH, a viscosidade e a presença de permeadores (LIMA EA, 2015).

Os efeitos colaterais de forma geral são mínimos, Saraiva LPPG, et al. (2018), no seu estudo das 17 voluntárias apenas duas ocorreu piora inicial após a segunda sessão, mas com relação ao componente vascular houve melhora após as sessões subsequentes. Mas observou-se surgimento de pápula eritematosa em uma voluntária que foi relacionada ao uso do creme cicatrizante, solucionado após a substituição do produto. Relacionando com as queixas álgicas durante o tratamento, todas as pacientes definiram como tolerável e nenhuma solicitou a interrupção do tratamento por esse motivo.

O grau de desconforto foi considerado tolerável por $70 \%$ das pacientes, e $30 \%$ informaram não ter sentido dor. Todos os pacientes relataram ter retornado suas atividades imediatamente após o procedimento (LIMA EVA, et al., 2013). Segundo Pitassi LHU, et al. (2019), apenas três pacientes em seu 
estudo apresentaram algum tipo de sintoma na região logo após o tratamento com roller. Entre eles, eritema com dor, ressecamento cutâneo excessivo com leve ardência.

A respeito de todos tratamentos terapêuticos, para o controle do melasma sempre é desafiante, principalmente por ser extremamente recorrente, refratário e com insatisfações estéticas ao paciente. Assim sendo, a procura por novas modalidades terapêuticas e suas associações apresentam como objetivo potencializar os efeitos clareadores e minimizar os efeitos sistêmicos indesejáveis. O microagulhamento é um método minimamente invasivo, incluindo neocolagênese e neoelastogenese, deixando a camada epidérmica praticamente intacta, demostrando eficaz na diminuição da pigmentação melânica epidérmica, bem como permite a entrega de ativos cutâneos despigmentantes (LIMA EVA, et al., 2013; LIMA EA, 2015; KALIL C, et al., 2017; SARAIVA LPPG, et al., 2018; PITASSI LHU, et al., 2019).

Considerando-se a literatura disponível, o microagulhamento pode ser usado como veículo de ativos, estímulo isolado no rejuvenescimento, tratamento de flacidez, e eliminação de rugas, correção de cicatrizes bem como na melhora de estrias recentes e antigas (LIMA EVA, et al., 2013).

\section{CONSIDERAÇÕES FINAIS}

De acordo com os dados revisados na literatura, o microagulhamento ou indução percutânea de colágeno é um tipo de tratamento muito promissor e efetivo apresentado como tratamento minimamente invasivo para promover melhora clínica e histológica do melasma, efetivamente em pacientes com fototipos elevados. E, quando associado a princípios ativos (Drug delivery), se mostrou bastante eficaz e versátil, otimizando os resultados devido ao aumento da permeabilidade do extrato córneo. A técnica de microagulhamento se mostra eficaz em diversos estudos e quando aplicado em algumas disfunções estéticas faciais, principalmente no melasma, seja pela permeação de ativos despigmentantes ou pela estimulação de colágeno proporcionando resultados satisfatórios, demonstrando-se também que é um procedimento bem tolerado com mínimos efeitos adversos e alto índice de satisfação das pacientes. Porém, existem poucos estudos na literatura que avaliem essa combinação, dessa forma se faz necessário a publicação de ensaios clínicos, com menores riscos de viés, para melhor elucidação das dúvidas que persistem sobre $o$ assunto.

\section{REFERÊNCIAS}

1. CASAVECHI AM, et al. A Utilização da Vitamina $C$ e do Peeling de Diamante no Tratamento do Melasma Facial: um estudo comparativo. São Paulo, 2015.

2. CÉLIA K, et al. Drug Delivery assistido por lasers: revisão. Surg Cosmet Dermatol. 2016;8(3):193-203.

3. KALIL C, et al. Microagulhamento: série de casos associados Drug delivery. Surgical \& Cosmetic Dermatology, vol. 9, núm. 1, 2017, pp. 96-99.

4. KLAYN AP, et al. Microagulhamento como agente potencializador da permeação de princípios ativos corporais no tratamento de lipodistrofia localizada: estudo de casos. In: ENCONTRO INTERNACIONAL DE PRODUÇÃO CIENTÍFICA CESUMAR - EPCC, 8, 2013, Maringá. Anais Eletrônicos... Maringá: Editora Cesumar, 2013. p. 1-5.

5. LIMA EA. Microagulhamento em melasma facial recalcitrante: uma série de 22 casos. An Bras Dermatol. 2015;90(6):917-9.

6. LIMA EVA, et al. Microagulhamento: estudo experimental e classificação da injúria provocada. Surgical \& Cosmetic Dermatology, Rio de Janeiro, v. 5, n. 2, p. 110-114, abr./jun. 2013.

7. LIMA AA, et al. Os Benefícios do Microagulhamento no Tratamento das Disfunções Estéticas. Revista Científica da FHO|UNIARARAS v. 3, n. 1/2015.

8. PITASSI LHU, et al. Avaliação histológica da redução do conteúdo melânico cutâneo após realização de microagulhamento na região anterior do colo. Surg Cosmet Dermatol. Rio de Janeiro v.11 n.1 jan-mar. 2019 p. 49-54.

9. PIATTI IL. Microagulhamento e fatores de crescimento. Revista Personalité, São Paulo, ano 16, n. 8, p. 22-25, 2013.

10. PURIM MSK, AVELAR SFM. Fotoproteção, melasma e qualidade de vida em gestantes. Rev. Bras. Ginecol. Obstet. Vol 34, 5. Rio de Janeiro May, 2012. 
11. SARAIVA LPPG, et al. Tratamento de melasma facial com associação do microagulhamento robótico e Drug delivery de ácido tranexâmico. Surg Cosmet Dermatol. Rio de Janeiro v.10 n.4 out-dez. 2018, p. 333-9.

12. SILVA SN. Microagulhamento com uso de ácido tranexâmico para o tratamento de melasma. Recife: $O$ Autor, 2018.

13. SILVEIRA, LL. Técnica de microagulhamento utilizados em alguns tratamentos estéticos. Recife: Ed. do Autor, 2017.

14. STEINER D, et al. Estudo da avaliação da eficácia do ácido tranexâmico tópico e injetável no tratamento do melasma. Surg Cosmet Dermatol. 2009;1(4):174-7.

15. TAGLIOLATTO $S$, et al. Uso da técnica de indução percutânea de colágeno no tratamento da hiperpigmentação pós-inflamatória. Surgical \& Cosmetic Dermatology, vol. 9, núm. 2, 2017, pp. 160-164. 